\title{
Determination of the optimal extrusion temperature of the PMMA optical fibers
}

\author{
Malwina Niedźwiedź ${ }^{1}$, Małgorzata Gil2*, Mateusz Gargol ${ }^{1}$, Wiesław Podkościelny ${ }^{2}$, Paweł Mergo ${ }^{2}$ \\ ${ }^{\text {I} D e p a r t m e n t ~ o f ~ P o l y m e r ~ C h e m i s t r y, ~ M a r i a ~ C u r i e-S k ł o d o w s k a ~ U n i v e r s i t y, ~ M a r i a ~ C u r i e-S k ł o d o w s k a ~ S q . ~ 5, ~ 20-031, ~}$ \\ Lublin, Poland \\ ${ }^{2}$ Laboratory of Optical Fibre Technology, Maria Curie-Sklodowska University, Maria Curie- Sklodowska Sq. 5, 20- \\ 031, Lublin, Poland
}

Received March 13, 2019; accepted March 28, 2019; published March 31, 2019

\begin{abstract}
The aim of this work was to determine the optimal extrusion temperature for polymer optical fibers. For preliminary studies poly(methyl methacrylate) (PMMA) granulate was used. The samples of commercially available PMMA were subjected to four different temperatures in which they were kept in an oven for three different periods of time. To examine the changes in the chemical structure of the polymer, ATR-FT-IR (Attenuation Total Reflection Fourier Transform Infrared Spectroscopy) was chosen.
\end{abstract}

Polymer optical fibers (POFs) are attractive materials for telecommunication and sensing applications. Their ease of handling, great flexibility and high resistance to external factors are the main advantages in these fields [1]. On the other hand, their relatively high signal attenuations according to silica optical fibers are a disadvantage for long-haul communications.

Attenuation can be proceeded according to two main mechanisms namely those of intrinsic and extrinsic loss. Intrinsic losses are directly related to the composition of an optical fiber and extrinsic losses are due to the absorption and dispersion caused by impurities and structural defects in POF, bending losses and connector losses. Bending loss is the result of fiber curvature from an ideal straight-line configuration [2]. Therefore, to produce good polymer optical fiber, it is very important to optimize the process of optical fiber drawing, as well the synthesis or/and purification of materials used. Thus, one of the main objectives of this work was to examine the influence of extrusion temperature on the chemical structure of polymer granulate from which future POFs will be fabricated.

The production of polymer optical fibers is a multi stage process. There are several different methods to fabricate POFs and each of them has its own advantages and disadvantages. There are some basic steps in the POF production like cleaning of raw materials and stretching.

A typical fiber fabrication technique is the heatdrawing process from a preform. This technique is well known from conventional glass fiber manufacturing, which relies on heating the preform in a furnace and

\footnotetext{
*E-mail: malgorzata.gil@ poczta.umcs.lublin.pl
}

drawing it to a fiber [3]. The second well-known method is extrusion from granulate. For some cases, it is easier to buy polymer granulate, which has some special properties, than synthesized it and purified [4].

This work is mainly focused on optimizing the second technique. In our studies we have chosen poly(methyl methacrylate) as a preliminary material. The main reason was its price and common use [5].

Samples of commercially available PMMA (Plexiglas, Darmstadt, Germany) were placed in test tubes and subjected to $90^{\circ} \mathrm{C}$ for $2 \mathrm{~h}$, to evaporate physically adsorbed water. The next step was heat treatment. The samples were submitted to four different temperatures: $180^{\circ} \mathrm{C}$, $210^{\circ} \mathrm{C}, 240^{\circ} \mathrm{C}, 270^{\circ} \mathrm{C}$, in which they were kept for three different periods of time: 20,40 and $60 \mathrm{~min}$ (in $270^{\circ} \mathrm{C}$ samples were kept also for 2 and $4 \mathrm{~h}$ ). The change in the chemical structure of tested samples was evaluated by using ATR-FT-IR (TENSOR 27 - Platinum ATR, Bruker). We chose this technique because it is fast (one analysis takes about $1 \mathrm{~min}$ ) and perfect for measuring polymers surface [6]. This method has a penetration depth of around 1 or 2 micrometers depending on the ATR crystal material. While doing the ATR-FT-IR analysis, for all samples, five analyses with 32 scans each were made. The FT-IR spectra were recorded in the spectral range of $700 \div 1900 \mathrm{~cm}^{-1}$ per spectrum at a resolution of $4 \mathrm{~cm}^{-1}$.

All the spectra were normalized and as reference the band $1720 \mathrm{~cm}^{-1}$ was chosen [7]. This band is related to a carbonyl group which does not change during the thermal treatment [8] (Fig.1). 


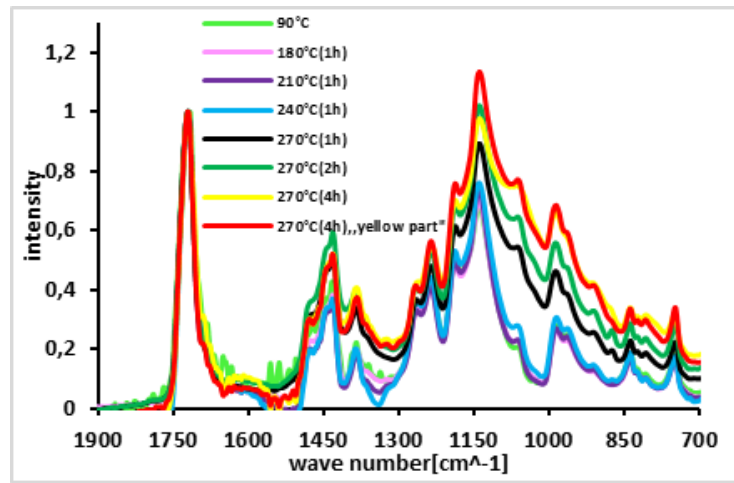

Fig. 1. PMMA ATR-FT-IR full spectra range.

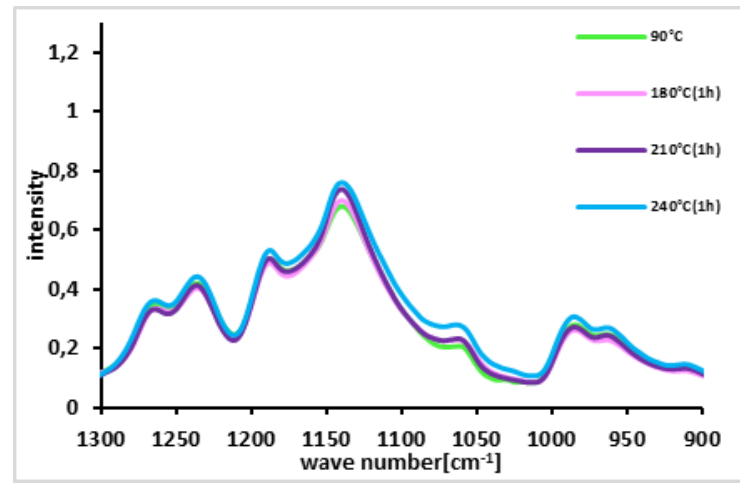

Fig. 2. PMMA ATR-FT-IR narrow spectra range

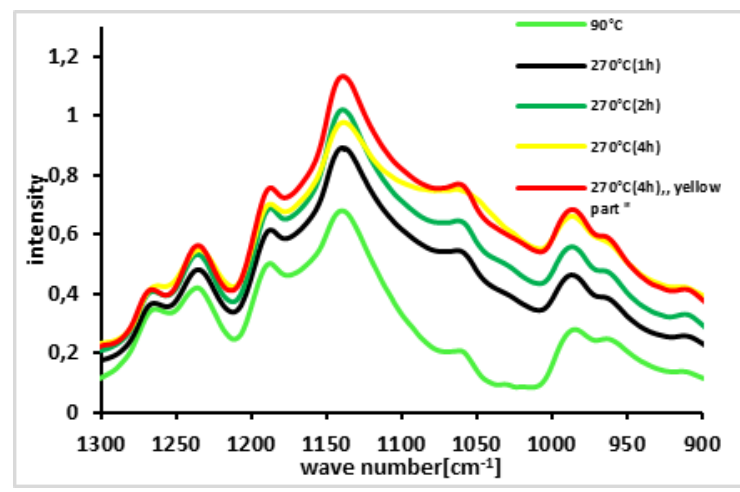

Fig. 3. PMMA ATR-FT-IR narrow spectra range

Relying on obtained results, it can be concluded that the maximum temperature at which POFs can be extruded is $240{ }^{\circ} \mathrm{C}$ (Tab. 1-5). Up to this temperature no change in the band intensity is visible, so the chemical structure of the tested polymer does not change (Fig.2). The only change can be observed for the band $1065 \mathrm{~cm}^{-1}$ related to $\mathrm{CH}_{3}$ rocking vibration. Doubling of this band intensity is already noticeable for the sample subjected to $240{ }^{\circ} \mathrm{C}$ for $1 \mathrm{~h}$, but the shape of all bands does not change. This can be explained as a result of post thermal polymerization.
Table 1. Band intensity measured in temperature $180^{\circ} \mathrm{C}$

\begin{tabular}{|c|c|c|c|c|c|}
\hline $\begin{array}{c}\text { Wave } \\
\text { number } \\
{\left[\mathrm{cm}^{-1}\right]}\end{array}$ & $\begin{array}{c}\text { Functional } \\
\text { group }\end{array}$ & $90^{\circ} \mathrm{C}$ & $\begin{array}{c}180^{\circ} \mathrm{C} \\
20 \mathrm{~min}\end{array}$ & $\begin{array}{c}180^{\circ} \mathrm{C} \\
40 \mathrm{~min}\end{array}$ & $\begin{array}{c}180^{\circ} \mathrm{C} \\
60 \mathrm{~min}\end{array}$ \\
\hline 810 & $\begin{array}{c}\text { Deformation } \\
\text { vibration } \\
=\mathrm{CH}\end{array}$ & 1.0 & 1.0 & 1.1 & 1.1 \\
\hline 989 & RHC=CH & 1.0 & 1.0 & 1.0 & 1.0 \\
\hline 1065 & $\begin{array}{c}\text {-CH3 } \\
\text { rocking } \\
\text { vibration }\end{array}$ & 1.0 & 1.4 & 1.2 & 1.2 \\
\hline 1142 & $\begin{array}{c}\text { Sym. } \\
\text { C-O-C } \\
\text { stretching } \\
\text { vibration }\end{array}$ & 1.0 & 1.0 & 1.0 & 1.1 \\
\hline 1190 & $\begin{array}{c}\text { Skeletal } \\
\text { chain }\end{array}$ & 1.0 & 1.0 & 1.0 & 1.0 \\
\hline
\end{tabular}

Table 2. Band intensity measured in temperature $210^{\circ} \mathrm{C}$

\begin{tabular}{|c|c|c|c|c|c|}
\hline $\begin{array}{c}\text { Wave } \\
\text { number } \\
{\left[\mathrm{cm}^{-1}\right]}\end{array}$ & $\begin{array}{c}\text { Functional } \\
\text { group }\end{array}$ & $90^{\circ} \mathrm{C}$ & $\begin{array}{c}210^{\circ} \mathrm{C} \\
20 \mathrm{~min}\end{array}$ & $\begin{array}{c}210^{\circ} \mathrm{C} \\
40 \mathrm{~min}\end{array}$ & $\begin{array}{c}210^{\circ} \mathrm{C} \\
60 \mathrm{~min}\end{array}$ \\
\hline 810 & $\begin{array}{c}\text { Deformation } \\
\text { vibration } \\
=\mathrm{CH}\end{array}$ & 1.0 & 1.0 & 1.0 & 1.0 \\
\hline 989 & $\mathrm{RHC}=\mathrm{CH}_{2}$ & 1.0 & 1.0 & 1.0 & 1.2 \\
\hline 1065 & $\begin{array}{c}-\mathrm{CH}_{3} \\
\text { rocking } \\
\text { vibration }\end{array}$ & 1.0 & 1.2 & 1.2 & 1.3 \\
\hline 1142 & $\begin{array}{c}\text { Sym. } \\
\text { C-O-C } \\
\text { stretching } \\
\text { vibration }\end{array}$ & 1.0 & 1.1 & 1.1 & 1.1 \\
\hline 1190 & $\begin{array}{c}\text { Skeletal } \\
\text { chain }\end{array}$ & 1.0 & 1.0 & 1.0 & 1.1 \\
\hline
\end{tabular}

Table 3. Band intensity measured in temperature $240^{\circ} \mathrm{C}$

\begin{tabular}{|c|c|c|c|c|c|}
\hline $\begin{array}{c}\text { Wave } \\
\text { number } \\
{\left[\mathrm{cm}^{-1}\right]}\end{array}$ & $\begin{array}{c}\text { Functional } \\
\text { group }\end{array}$ & $90^{\circ} \mathrm{C}$ & $\begin{array}{c}240^{\circ} \mathrm{C} \\
20 \mathrm{~min}\end{array}$ & $\begin{array}{c}240^{\circ} \mathrm{C} \\
40 \mathrm{~min}\end{array}$ & $\begin{array}{c}240^{\circ} \mathrm{C} \\
60 \mathrm{~min}\end{array}$ \\
\hline 810 & $\begin{array}{c}\text { Deformation } \\
\text { vibration } \\
=\mathrm{CH}\end{array}$ & 1.0 & 1.0 & 1.2 & 1.2 \\
\hline 989 & RHC=CH 2 & 1.0 & 1.0 & 1.2 & 1.4 \\
\hline 1065 & $\begin{array}{c}\text {-CH} \\
\text { rocking } \\
\text { vibration }\end{array}$ & 1.0 & 1.2 & 1.6 & 2.1 \\
\hline 1142 & $\begin{array}{c}\text { Sym. } \\
\text { C-O-C } \\
\text { stretching } \\
\text { vibration }\end{array}$ & 1.0 & 1.0 & 1.2 & 1.2 \\
\hline 190 & $\begin{array}{c}\text { Skeletal } \\
\text { chain }\end{array}$ & 1.0 & 1.0 & 1.2 & 1.2 \\
\hline
\end{tabular}


Table 4. Band intensity measured in temperature $270^{\circ} \mathrm{C}$

\begin{tabular}{|c|c|c|c|c|c|}
\hline $\begin{array}{c}\text { Wave } \\
\text { number } \\
{\left[\mathrm{cm}^{-1}\right]}\end{array}$ & $\begin{array}{c}\text { Functional } \\
\text { group }\end{array}$ & $90^{\circ} \mathrm{C}$ & $\begin{array}{c}270^{\circ} \mathrm{C} \\
20 \mathrm{~min}\end{array}$ & $\begin{array}{c}270^{\circ} \mathrm{C} \\
40 \mathrm{~min}\end{array}$ & $\begin{array}{c}270^{\circ} \mathrm{C} \\
60 \mathrm{~min}\end{array}$ \\
\hline 810 & $\begin{array}{c}\text { Deformation } \\
\text { vibration } \\
=\mathrm{CH}\end{array}$ & 1.0 & 1.4 & 1.7 & 1.4 \\
\hline 989 & $\mathrm{RHC}=\mathrm{CH}_{2}$ & 1.0 & 1.6 & 1.8 & 1.6 \\
\hline 1065 & $\begin{array}{c}-\mathrm{CH}_{3} \\
\text { rocking } \\
\text { vibration }\end{array}$ & 1.0 & 2.4 & 2.9 & 2.7 \\
\hline 1142 & $\begin{array}{c}\text { Sym. } \\
\text { C-O-C } \\
\text { stretching } \\
\text { vibration }\end{array}$ & 1.0 & 1.2 & 1.3 & 1.2 \\
\hline 1190 & $\begin{array}{c}\text { Skeletal } \\
\text { chain }\end{array}$ & 1.0 & 1.2 & 1.2 & 1.1 \\
\hline
\end{tabular}

Table 5. Band intensity measured in temperature $270^{\circ} \mathrm{C}$

\begin{tabular}{|c|c|c|c|c|}
\hline $\begin{array}{c}\text { Wave } \\
\text { number } \\
{\left[\mathrm{cm}^{-1}\right]}\end{array}$ & $\begin{array}{c}\text { Functional } \\
\text { group }\end{array}$ & $90^{\circ} \mathrm{C}$ & $\begin{array}{c}270^{\circ} \mathrm{C} \\
2 \mathrm{~h}\end{array}$ & $\begin{array}{c}270^{\circ} \mathrm{C} 4 \mathrm{~h} \\
\text { "yellow } \\
\text { part" }\end{array}$ \\
\hline 810 & $\begin{array}{c}\text { Deformation } \\
\text { vibration } \\
=\mathrm{CH}\end{array}$ & 1.0 & 1.5 & 1.6 \\
\hline 989 & $\mathrm{RHC}=\mathrm{CH}_{2}$ & 1.0 & 1.8 & 2.0 \\
\hline 1065 & $\begin{array}{c}-\mathrm{CH}_{3} \\
\text { rocking } \\
\text { vibration }\end{array}$ & 1.0 & 3.4 & 3.6 \\
\hline 1142 & $\begin{array}{c}\text { Sym. } \\
\text { C-O-C } \\
\text { stretching } \\
\text { vibration }\end{array}$ & 1.0 & 1.3 & 1.4 \\
\hline 1190 & $\begin{array}{c}\text { Skeletal } \\
\text { chain }\end{array}$ & 1.0 & 1.2 & 1.4 \\
\hline
\end{tabular}

As can be seen in Fig. 3 and Tables $4 \div 5$, the bands intensity and shape of the spectrum change significantly. An over threefold increase can be observed for the band $1065 \mathrm{~cm}^{-1}$, in the "yellow part" of the sample subjected to $270^{\circ} \mathrm{C}$ for $4 \mathrm{~h}$. The "yellow part" should be understood as the upper part of the sample, which has changed color during heat treatment.

For obtained results, it was possible to calculate the double band conversion (Tab. 6). The double band conversion value indicates the amount of reacted monomer, which is crucial because it affects the quality grade and thermal stability of a polymer. Double band conversion was calculated according to the equation:

$$
\pi=\left[1-\left(\frac{\mathrm{A}_{1720 \text { monomer }}}{\mathrm{A}_{810 \text { monomer }}}\right) *\left(\frac{\mathrm{A}_{810} \text { polymer }}{\mathrm{A}_{1720 \text { polvmer }}}\right)\right] * 100 \%
$$

where:

$\mathrm{A}_{1720 \text { monomer }}$-the band absorbance at the wavelength $1720 \mathrm{~cm}^{-1}$ derived from the monomer,

$\mathrm{A}_{810 \text { monomer }}$-the band absorbance at the wavelength $810 \mathrm{~cm}^{-1}$ derived from the monomer,

$\mathrm{A}_{810 \text { polymer }}$-the band absorbance at the wavelength $810 \mathrm{~cm}^{-1}$ derived from the polymer,

$\mathrm{A}_{1720 \text { polymer }}$-the band absorbance at the wavelength $1720 \mathrm{~cm}^{-1}$ derived from the polymer,

$\pi$-the double bond conversion
Table 6. Double bond conversion

\begin{tabular}{|l|l|l|l|l|}
\hline & \multicolumn{2}{|l|}{ Time [min] } & Time [h] \\
\hline & 20 & 40 & 60 & 4 \\
\hline $90{ }^{\circ} \mathrm{C}$ & 89.6 & 89.3 & 89.4 & - \\
\hline $180^{\circ} \mathrm{C}$ & 89.6 & 90.3 & 90.4 & - \\
\hline $210^{\circ} \mathrm{C}$ & 90.6 & 89 & 89.6 & - \\
\hline $240^{\circ} \mathrm{C}$ & 90.7 & 82.8 & 85.8 & 84.7 \\
\hline $270^{\circ} \mathrm{C}$ & 86.2 & \multicolumn{5}{|l|}{} \\
\hline
\end{tabular}

As is shown in Table 6, the value of double bond conversion, obtained for $20 \mathrm{~min}$, increases with the temperature increase (till $240^{\circ} \mathrm{C}$ ). It may be the result of post thermal polymerization. In the case of longer heating this change is not noticeable.

In $270^{\circ} \mathrm{C}$ the double bond conversion value decreases. This can indicate depolymerization as well as thermal degradation [9] of the tested material. Depolymerization is also confirmed by an increase in the intensity of bands: $810 \mathrm{~cm}^{-1}$, related to deformation vibration $=\mathrm{CH}$ and

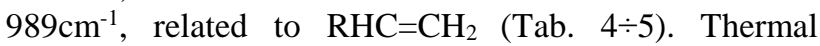
degradation is confirmed by an increase in the intensity of bands, from the same tables, e.g. band $1065 \mathrm{~cm}^{-1}$. Therefore, no process can be excluded, most likely both occur.

Based on the ATR-FT-IR spectra, it was deduced that the chemical structure of PMMA above $240^{\circ} \mathrm{C}$ is thermally changing. The ratio of spectral bands intensity increased with thermal increasing. $240^{\circ} \mathrm{C}$ is the highest temperature at which optical fibers, made from PMMA granulate, can be drawn. Till $240^{\circ} \mathrm{C}$, double bond conversion values do not change. This means that probably the PMMA structure does not change. In higher temperature this value decreases, which means that the polymer degrades or/and depolymerises.

\section{References}

[1] K. Peters, Smart Mater. Struct. 20, 2281 (2010).

[2] O. Ziemann, J. Krauser, P.E. Zamzow, W. Daum, POF Polymer Optical Fibers for Data Communication (New York, Springer-Verlag Berlin Heidelberg 2002).

[3] M.A. van Eijkelenborg, M.C.J. Large, A. Argyros, J. Zagari, S. Manos, N.A. Issa, I. Bassett, S. Fleming, R.C. McPhedran, C. Martijn de Sterke, N.A.P. Nicorovici, Opt. Expr. 9, 319 (2001).

[4] O. Çetinkaya, G. Wojcik, P. Mergo, Mater. Res. Expr. 5, 1 (2018).

[5] P. Mergo, M. Gil, K. Skorupski, J. Klimek, G. Wójcik, J. Pędzisz, J. Kopec, K. Poruraj, L. Czyzewska, A. Walewski, A. Gorgol, Phot. Lett. Poland 5, 170 (2013)

[6] J. Grdadolnik, Acta Chim Slov. 49, 631 (2002).

[7] P. Borowski, S. Pasieczna-Patkowska, M. Barczak, K. Pilorz, J. Phys. Chem. A 116, 7424 (2012).

[8] G. Socrates, Infrared and Raman Characteristic Group Frequencies Tables and Charts (Third Edition Baffins Lane Chichester, John Wiley \& Sons Ltd 2001).

[9] W. Schnabel, Polymer Degradation Principles and Practical Applications (Berlin, Akademie-Verlag 1981). 